\title{
Togitare
}

\section{INTERVENÇÕES BREVES JUNTO A UTENTES DA ATENÇÃO PRIMÁRIA EM USO DE RISCO E NOCIVO DE ÁLCOOL}

Fernanda Matos Fernandes Castelo Branco1, Ana Cristina Pais Abreu Ferreira², Tereza Maria Mendes Diniz de Andrade Barroso ${ }^{3}$

\section{RESUMO}

Objetivo: avaliar o efeito das intervenções breves na redução do consumo de álcool em utentes de uma Unidade de Saúde Familiar.

Método: estudo pré-experimental, avaliação antes e depois (quatro meses) de 205 utentes (grupo único). Utilizou-se o Alcohol Use Disorders ldentification Test(AUDIT). A coleta ocorreu em Coimbra, Portugal, nos meses de maio/junho e setembro/outubro de 2019. Utilizou-se o teste Wilcoxon para avaliação dos efeitos das intervenções.

Resultados: na primeira avaliação, 189 (92,2\%) encontravam-se na zona I; 15 (7,3\%) na zona II e $1(0,5 \%)$ na zona IV. No seguimento, quatro meses após as intervenções com os 15 utentes que pontuaram zona 2, teve-se perda da amostra de 5, demonstrando que $6(60 \%)$ pontuaram zona I, $3(30 \%)$ zona ll e 1 (10\%) zona III.

Conclusão: as intervenções breves são recursos efetivos na detecção precoce do consumo de álcool, sendo necessários à disseminação nos cuidados primários à saúde.

DESCRITORES: Alcoolismo; Consumo de Bebidas Alcoólicas; Serviços Preventivos de Saúde; Atenção Primária à Saúde; Avaliação de Eficácia-Efetividade de Intervenções.

COMO REFERENCIAR ESTE ARTIGO:

Branco FMFC, Ferreira ACPA, Barroso TMMD de A. Intervenções breves junto a utentes da atenção primária em uso de risco e nocivo de álcool. Cogitare enferm. [Internet]. 2020 [acesso em "colocar data de acesso, dia, mês abreviado e ano"]; 25. Disponível em: http://dx.doi.org/10.5380/ce.v25i0.73502.

${ }^{1}$ Enfermeira. Pós-doutora em Enfermagem. Docente da Universidade Federal do Amapá. Oiapoque, AP, Brasil. (1) ${ }^{2}$ Enfermeira. Especialista em Enfermagem Comunitária. Enfermeira da Unidade de Saúde Familiar Rainha Santa Isabel. Coimbra, Portugal. (D)

${ }^{3}$ Enfermeira. Pós-doutora em Enfermagem. Docente da Escola Superior de Enfermagem/Unidade de Investigação em Ciências da Saúde. Coimbra, Portugal. (iD 


\title{
BRIEF INTERVENTIONS FOR PRIMARY CARE USERS ON RISKY AND HARMFUL USE OF ALCOHOL
}

\begin{abstract}
Objective: to evaluate the effect of brief interventions in decreasing alcohol use in users of a Family Health Unit.

Method: a pre-experimental study, before and after evaluation (four months) of 205 users (single group). The Alcohol Use Disorders Identification Test (AUDIT) was used. Data collection took place in Coimbra, Portugal, in May/June and September/October 2019. The Wilcoxon test was used to assess the effects of the interventions.

Results: in the first assessment, 189 (92.2\%) were in zone I; 15 (7.3\%) in zone II and 1 (0.5\%) in zone IV. In the follow-up, four months after the interventions with the 15 users who scored zone 2, there was a sample loss of 5, showing that $6(60 \%)$ scored zone I, $3(30 \%)$ zone II and 1 (10\%) zone III.

Conclusion: brief interventions are effective resources in the early detection of alcohol use, which are necessary for dissemination in primary health care.
\end{abstract}

DESCRIPTORS: Alcoholism; Alcohol Drinking; Preventive Health Services; Primary Health Care; valuation of the Efficacy-Effectiveness of Interventions.

\section{INTERVENCIONES BREVES CON PACIENTES DE ATENCIÓN PRIMARIA EN USO DE RIESGO Y NOCIVO DE ALCOHOL}

\section{RESUMEN:}

Objetivo: Evaluar el efecto de intervenciones breves en la reducción de ingesta alcohólica de usuarios de una Unidad de Salud Familiar.

Método: Estudio preexperimental, evaluación antes y después (cuatro meses) de 205 usuarios (grupo único). Se utilizó Alcohol Use Disorders Identification Test (AUDIT). Datos recolectados en Coímbra, Portugal, en mayo/junio y setiembre/octubre de 2019. Se aplicó test Wilcoxon para evaluar el efecto de las intervenciones.

Resultados: En la primera evaluación, 189 (92,2\%) estaba en zona I; 15 (7,3\%) en zona II y 1 $(0,5 \%)$ en zona IV. En el seguimiento, cuatro meses después de las intervenciones con los 15 usuarios de zona II, se observó pérdida de la muestra de 5, expresando que 6 (60\%) puntuaron como zona I, 3 (30\%) zona II; y 1 (10\%), zona III.

Conclusión: Las intervenciones breves constituyen recursos efectivos para detección precoz del consumo alcohólico, siendo necesaria su diseminación en atención primaria de salud.

DESCRIPTORES: Alcoholismo; Consumo de Bebidas Alcohólicas; Servicios Preventivos de Salud; Atención Primaria de Salud; Evaluación de Eficacia-Efectividad de Intervenciones. 
O consumo excessivo de álcool é considerado um problema de saúde pública mundial, causando graves consequências em nível individual e coletivo. Segundo o relatório da Organização Mundial de Saúde (OMS), no ano de 2016, o uso nocivo de álcool resultou em cerca de 3 milhões de mortes (5,3\% das mortes no mundo inteiro). A mortalidade resultante do consumo de álcool é maior do que as mortes oriundas de doenças como a tuberculose, HIV/AIDS e diabetes ${ }^{(1)}$. Embora sejam evidenciados riscos à saúde da população mundial devido ao abuso de álcool, é difícil pensar na sua extinção ao longo da vida, sendo, portanto, um problema que concerne milhões de pessoas, sem distinção cultural(2).

Em Portugal, de acordo com o Relatório do ano de 2018 relativo aos padrões de consumo abusivo e dependência de álcool, $2,8 \%$ da população (4,9\% dos consumidores) tinha, no último ano pesquisado, um consumo considerado de risco elevado/nocivo e $0,8 \%$ $(1,3 \%$ dos consumidores) apresentavam sintomas de dependência, conforme o Alcohol Use Disorders Identification Test (AUDIT) ${ }^{(3)}$.

Diante dessa conjuntura e pensando no processo de cuidar, destacam-se as Intervenções Breves (IBs) relacionadas ao consumo de risco e nocivo de álcool e outras drogas, tanto devido à sua universalidade quanto à grande proporção da população que acessa os serviços de saúde diariamente.

A intervenção breve é um tipo de atendimento com tempo limitado, cujo foco é a mudança de comportamento do paciente. São estabelecidas algumas etapas: avaliação do problema (triagem), devolutiva (feedback), estabelecimento de metas, discussão de prós e contras do uso, aconselhamento e desenvolvimento de autoeficácia do paciente. Usa uma abordagem de redução de danos, buscando diminuição de comportamento de risco e efeitos prejudiciais da droga, para as pessoas que consomem de forma abusiva e nociva ${ }^{(4)}$.

A técnica da IB pode ser aplicada por profissionais de várias formações, desde que tenham recebido um breve treinamento. Desde simples recomendações para redução do consumo, fornecidas por um profissional, até uma série de recursos oferecidos em um programa estruturado de tratamento podem ser empregados ${ }^{(5)}$.

A formação de profissionais de saúde, especialmente os enfermeiros, é essencial para a detecção precoce dos consumos de risco de álcool; implementar as Intervenções Breves na redução de consumo excessivo de álcool, monitorização e encaminhamento, deve, portanto, ser uma prioridade durante o processo de formação profissional(6).

É necessário valorizar aspectos da formação em enfermagem, não se limitando apenas aos conhecimentos técnicos, mas enaltecer os recursos existentes para um cuidado integral, com implicações positivas na assistência às pessoas que consomem álcool e outras drogas de forma abusiva ${ }^{(7)}$. Desta via, percebe-se a relevância da aplicação das IBs nos cuidados primários de saúde, sendo os enfermeiros peças fundamentais no processo da detecção precoce de utentes com padrões de risco/nocivo em relação ao uso de álcool e a apliçação das IBs de acordo com o nível de risco encontrado(8).

Para se alcançar um modelo de cuidar voltado para a promoção da saúde e prevenção de agravos, os profissionais envolvidos devem estar preparados e aptos a reconhecer os problemas relacionados ao uso de drogas e desenvolver ações assistenciais. E considerando o estudo(9) que aponta os serviços de cuidado primários de saúde como porta de entrada dos usuários, familiares e comunidades em geral, pode-se afirmar que estes são espaços relevantes para realizações de formas de cuidar e tratar.

No Brasil, a atuação dos enfermeiros na Estratégia Saúde da Família (ESF) é essencial na identificação dos pacientes, bem como na sistematização da assistência na atenção primária, conforme preconizado nas atuais políticas de saúde nacionais e internacionais ${ }^{(10)}$. 
Destarte, no intuito de compreender o consumo de risco/nocivo, são imperativas estratégias e intervenções mais condizentes com os utentes que usam bebidas alcóolicas. É também necessário que os programas de atendimento e as políticas desenvolvidas sejam mais embasadas nas evidências científicas já disponíveis sobre o tratamento das dependências químicas, para que sejam mais efetivas e haja maior controle deste grave problema de saúde pública. Todavia, as IBs não estão disseminadas nas práticas clínicas e os estudos acerca desse domínio são escassos.

Salienta-se que em Portugal existem diretrizes da Direção Geral de Saúde que recomendam a utilização das IBs, através na Norma 030/2012 de 28 de dezembro, atualizada em 18/12/2014(11). Nesse contexto, sobreleva-se a importância desse estudo. Especula-se que as implicações para a prática assistencial ocorrerão no sentido de contribuir para que órgãos e entidades que tratam da saúde populacional, governamentais ou não, possam elaborar estratégias e políticas voltadas para o aprimoramento da saúde dessa população, especificamente no que se refere ao uso de álcool, proporcionando melhorias na qualidade de vida e prevenindo agravos decorrentes do uso problemático de álcool.

Portanto, este estudo objetivou avaliar o efeito das Intervenções Breves na redução do consumo de álcool em utentes de uma Unidade de Saúde Familiar.

\section{MÉTODO}

Trata-se de um estudo de natureza quantitativa, pré-experimental, de avaliação antes e após as IBs (quatro meses após), com grupo único. O estudo foi realizado na Unidade de Saúde Familiar (USF) Rainha Santa Isabel, nos Polos assistenciais Santa Clara, Cernache e Almalaguês, localizados em Coimbra - Portugal, com amostra por conveniência, constituída pelos utentes cadastrados na Unidade de Saúde. A coleta ocorreu nos meses de maio e junho-2019 e após quatro meses (setembro/outubro) do mesmo ano. A escolha para este tempo se deu baseado em estudo ${ }^{(12)}$ que orienta avaliação seguinte após quatro a seis meses.

A amostra foi constituída por 205 utentes, que tinham mais de 18 anos, disponibilidade e aceitaram o consentimento formal para responder ao inquérito, sendo estes os critérios de inclusão. Para o pós-teste, foram considerados critérios de exclusão: utentes que pontuaram zona IV, ou seja, provável dependência e que apresentavam alguma deficiência ou incapacidade de responder aos questionamentos.

Para a coleta de dados, utilizou-se um questionário sociodemográfico e o Alcohol Use Disorder Test (AUDIT), questionário de avaliação de níveis de risco para o consumo de álcool indicado pela norma Clínica da Direção Geral de Saúde de Portugal.

O AUDIT é um instrumento de rastreio, reconhecido mundialmente, desenvolvido pela OMS, para avaliação de risco relativo ao consumo de álcool, em serviços de saúde de diferentes níveis e contextos. É um questionário composto por 10 questões, que abordam três domínios (padrão do consumo de álcool, sinais e sintomas de dependência e problemas decorrentes do uso de álcool) e cujas pontuações variam identificando quatro padrões de uso: consumo que não levará a problemas: 0 a 7 pontos (baixo risco - zona 1), receberam uma intervenção educacional; consumo que poderá levar a problemas: 8 a 15 pontos (uso de risco - zona 2), receberam aconselhamento simples; consumo que provavelmente poderá levar a problemas: 16 a 19 pontos (uso nocivo - zona 3), receberam aconselhamento simples, aconselhamento breve e acompanhamento contínuo e consumo que provavelmente já origina problemas: 20 pontos ou mais (possível dependência - zona 4), foram referenciados para diagnóstico, avaliação, e tratamento(4).

Os três últimos padrões de uso são tidos como uso problemático, apresentando este instrumento boa especificidade e sensibilidade para detectar os diferentes padrões 
nestes casos. As IBs foram desenvolvidas com base em guias estruturadas, adaptadas ${ }^{(4)}$, no âmbito do projeto Saúde sem Reservas inscrito na Unidade de Investigação em Ciências da Saúde: Enfermagem.

Os dados foram coletados por enfermeiros capacitados, especialistas em saúde mental com treino específico, através de entrevistas em local privado e reservado. Os questionários foram numerados e codificados para posterior avaliação no pós teste, no qual cada utente recebeu um cartão com as informações de contato telefônico e data de agendamento do pós teste. $\mathrm{Na}$ fase do rastreio, foi identificado um utente com provável dependência que foi referenciado para diagnóstico, e não integrou a avaliação posterior. Foi obtido consentimento informado e esclarecido de todos os participantes.

Para a análise dos dados adquiridos, recorreu-se ao uso do software Statistical Package for the Social Sciences (SPSS), versão 22.0. Foram utilizados os testes de normalidade Shapiro-Wilk (para uma amostra inferior a 50 sujeitos), todas as variáveis dependentes põem em causa os pressupostos da normalidade, decorrente do tipo de variável em estudo (níveis de risco para o consumo de álcool) e do tipo de amostra (amostra emparelhada), optou-se pelo teste não paramétrico Wilcoxon, para avaliação do efeito das IBs.

Para atender aos critérios éticos, a pesquisa foi submetida ao Comitê de Ética em Pesquisa da Escola Superior de Enfermagem de Coimbra, da Unidade de Investigação em Ciências da Saúde (UICISA:E), com parecer P550/01-2019. Antes do processo de coleta dos dados, obteve-se consentimento do Conselho Técnico de Administração da Unidade de Saúde Familiar Rainha Santa Isabel, na sequência autorização da Administração Regional de Saúde Centro, pertencente ao governo de Portugal, com parecer 120/23.

\section{RESULTADOS}

A amostra foi constituída por 205 indivíduos, sendo a média de idades de 55,2 anos (DP=17,1). Quanto ao sexo, observou-se predomínio do feminino ( $n=120 / 58,5 \%)$. Relativamente à escolaridade, verificou-se que 60 entrevistados $(29,7 \%)$ da amostra tem o $1^{\circ}$ ciclo; $148(74,4 \%)$ são casados, $83(42,1 \%)$ são reformados, $167(81,5 \%)$ vivem em casa própria, $186(91,2 \%)$ possuem rendimento (Tabela 1).

Tabela 1 - Características socieconômicas dos utentes da Unidade de Saúde Familiar Rainha Santa Isabel. Coimbra, Portugal, 2019 (continua)

\begin{tabular}{lcc} 
Variáveis & $\mathbf{n}(\%)^{*}$ & $\%$ \\
\hline Sexo & & \\
\hline Masculino & 85 & 41,5 \\
\hline Feminino & 120 & 58,5 \\
\hline Faixa Etária & & \\
\hline$<20$ anos & 2 & 1 \\
\hline $20|-| 59$ anos & 107 & 52,2 \\
\hline$\geq 60$ anos & 96 & 46,8 \\
\hline Estado Civil & & \\
\hline Casado(a) /União de Facto & 148 & 74,4 \\
\hline Divorciado(a) /Separado(a) & 13 & 6,5 \\
\hline
\end{tabular}




\begin{tabular}{|c|c|c|}
\hline Solteiro(a) & 25 & 12,6 \\
\hline Viúvo(a) & 13 & 6,5 \\
\hline \multicolumn{3}{|l|}{ Habilitações Literárias } \\
\hline Não sabe ler e escrever & 1 & 0,5 \\
\hline$<4$ anos de escolaridade & 7 & 3,5 \\
\hline $1^{\circ}$ Ciclo ( $4^{\circ}$ ano) & 60 & 29,7 \\
\hline $2^{\circ}$ Ciclo (6 ano) & 6 & 3 \\
\hline $3^{\circ}$ Ciclo (9 ano) & 36 & 17,8 \\
\hline Bacharelato e Licenciatura & 51 & 25,2 \\
\hline Ensino Secundário $\left(10^{\circ}, 11^{\circ}, 12^{\circ}\right)$ & 41 & 20,3 \\
\hline \multicolumn{3}{|l|}{ Situação em que vive } \\
\hline Casa alugada & 36 & 17,6 \\
\hline Casa arrendada & 1 & 0,5 \\
\hline Casa da sogra & 1 & 0,5 \\
\hline Casa própria & 167 & 81,5 \\
\hline \multicolumn{3}{|l|}{ Tipo de ocupação } \\
\hline Desempregado(a) & 6 & 3 \\
\hline Empregado(a) & 65 & 33 \\
\hline Outro & 43 & 21,8 \\
\hline Reformado(a) & 83 & 42,1 \\
\hline \multicolumn{3}{|l|}{ Fonte de Rendimento } \\
\hline Com rendimento & 186 & 91,2 \\
\hline Sem rendimento & 18 & 8,8 \\
\hline
\end{tabular}

*Algumas colunas não pontuam os 205, pela falta de respostas de alguns participantes aos devidos questionamentos

Analisando o nível de risco, verificou-se que, na primeira avaliação, 189 (92,2\%) dos participantes apresentavam nível de risco I (baixo risco), 15 (7,3\%) estavam no nível de risco II (nível de risco) e um (0,5\%) no nível IV (provável dependência), este foi excluído e referenciado para o médico de família, sendo efetuado diagnóstico e encaminhamento para unidade especializada (Tabela 2).

Tabela 2 - Caracterização da classificação do AUDIT inicial dos utentes da Unidade de Saúde Familiar Rainha Santa Isabel. Coimbra, Portugal, 2019

\begin{tabular}{lcc} 
Níveis de riscos & $\mathbf{n}(\%)$ & Média \pm DP \\
\hline Zona I & $189(92,2)$ & \\
\hline Zona II & $15(7,3)$ & \\
\hline Zona IV & $1(0,5)$ & \\
\hline Escore AUDIT & & $3,0 \pm 3,4$
\end{tabular}


No seguimento, quatro meses após as intervenções com os 15 utentes que pontuaram Zona II, verificou-se uma perda da amostra de 5 . Dos 10 restantes, seis (60\%) pontuaram zona I, três (30\%) zona II e um (10\%) zona III (Tabela 3).

Tabela 3 - Relação da amostra antes e após IB quanto às zonas de risco dos utentes da Unidade de Saúde Familiar Rainha Santa Isabel. Coimbra, Portugal, 2019

\begin{tabular}{lcc} 
Nível de risco & $\begin{array}{c}\text { Pré-Intervenção } \\
\mathbf{n}(\%)\end{array}$ & $\begin{array}{c}\text { Pós-Intervenção } \\
\mathbf{n}(\%)\end{array}$ \\
\hline Zona I & $0(0)$ & $6(60)$ \\
\hline Zona II & $15(100)$ & $3(30)$ \\
\hline Zona III & $0(0)$ & $1(10)$ \\
\hline Zona IV & $0(0)$ & $0(0)$
\end{tabular}

A Tabela 4 aponta o teste de normalidade, Shapiro-Wilk. Com base no teste de normalidade, ao nível de significância de $5 \%$, temos evidência estatística que o escore do AUDIT no primeiro momento não segue distribuição normal, e o segundo momento segue uma distribuição normal.

Tabela 4 - Teste de Normalidade para os escores do questionarário AUDIT dos utentes da Unidade de Saúde Familiar Rainha Santa Isabel. Coimbra, Portugal, 2019

\begin{tabular}{lccc} 
& \multicolumn{3}{c}{ Shapiro-Wilk } \\
\cline { 2 - 4 } & Estatística & df & p-valor \\
\hline Escore AUDIT $\left(1^{\circ}\right.$ Momento) & 0,795 & 10 & 0,013 \\
\hline Escore AUDIT $\left(2^{\circ}\right.$ Momento) & 0,859 & 10 & 0,074 \\
$\star$ Teste de Normalidade, Shapiro-Wilk, ao nível de significância de $0,05(5 \%)$.
\end{tabular}

Por se tratar de uma pequena amostra, utilizamos na sequência o teste não paraméricos Teste de Wilcoxon.

Nos resultados apresentados (antes e após as IBs), e conforme se pode observar na Tabela 5, inerentes à evolução do nível de risco, verifica-se uma evolução positiva do nível de risco, ou seja, o nível de risco após a intervenção é menor que antes da intervenção. Há apenas um participante da amostra que aumentou o nível de risco, e três participantes mantiveram o nível de risco após a intervenção. Esta evolução positiva, como se pode verificar, indica um efeito positivo das IBs na redução do consumo de risco. 
Tabela 5 - Evolução da amostra relativamente à zona de risco, antes e após IB dos utentes da Unidade de Saúde Familiar Rainha Santa Isabel. Coimbra, Portugal, 2019

\begin{tabular}{llccc} 
& Postos & Frequência & Z & p-valor \\
\hline Escore AUDIT (2 Momento) / & Negativas & $7^{\mathrm{a}}$ & $-1,402$ & 0,161 \\
Escore AUDIT (1 & & & & \\
\hline & Positivas & $1^{\mathrm{b}}$ & & \\
\hline & Empate & $2^{\mathrm{c}}$ &
\end{tabular}

*Teste de Wilcoxon, ao nível de significância de $5 \%$.

Nota: a Nível de risco após < Nível de risco antes. b Nível de risco após > Nível de risco antes. c Nível de risco após = Nível de risco antes.

\section{DISCUSSÃO}

Mesmo diante de uma amostra reduzida, os resultados sugerem que os utentes submetidos a IB apresentam uma evolução positiva quanto aos níveis de consumo, reduzindo assim os níveis de risco. Fato que merece destaque e relevante para a prática clínica, foi o encaminhamento de um utente com provável dependência para consulta especializada devido a detecção precoce, que demonstrou prontidão para mudança, juntamente com apoio familiar.

Nessa conjuntura, estudo(13) que objetivou caracterizar a produção do conhecimento científico sobre a implementação de IB na atenção primária à saúde e identificar as dificuldades da equipe de saúde sobre o tema, constatou que a capacitação profissional de saúde para triagem do uso de álcool e a realização de IB são essenciais e necessárias. Entretanto, os autores apontam as dificuldades da equipe relacionadas ao planejamento, organização do sistema de saúde, crenças e atitudes dos trabalhadores voltadas aos usuários de álcool e a técnica aplicada, sendo necessária a ampliação de discussões acerca da temática, para que políticas públicas sejam incentivadas nos contextos dos serviços de saúde ${ }^{(13)}$.

No decorrer das entrevistas, verificou-se por alguns momentos reações de surpresas quando questionados os hábitos de consumo, o que pode estar associado à ausência de tais indagações durante consultas rotineiras nos serviços de saúde. Nessa vertente, estudiosos $^{(14)}$ apontam que os profissionais da atenção primária à saúde devem utilizar o Alcohol Smoking and Substance Involvement Screening Test (ASSIST) como ferramenta de rastreio precoce do consumo de álcool, a fim de intervir de forma breve, para evitar a busca por serviços secundários e terciários em decorrências de problemas relacionados ao uso abusivo de substâncias psicoativas.

Estudo que avaliou o padrão do consumo de álcool e suas consequências após a aplicação da intervenção BASICS (Brief Alcohol Screnning and Intervention for College Students), em uma amostra de universitários brasileiros, demonstrou a importância da IB e aplicação de instrumentos de rastreio, pontuando resultados positivos e efetivos da IB para diminuição do consumo problemático(15). Pesquisa também realizada entre universitários consumidores de risco de bebidas alcóolicas apontou que a IB apresentou resultado positivo, significativo e prontidão para mudança relacionado ao padrão do consumo, promovendo saúde ${ }^{(16)}$.

Contextualizando o efeito da IB nos serviços primários à saúde, pesquisa que verificou a efetividade da intervenção grupal realizada por enfermeiros na redução do uso de risco/ nocivo de álcool foi efetiva, sugerindo que a técnica seja replicada em outros serviços, pois 
demanda menor tempo dos profissionais de saúde a fim de atender um maior número de pacientes quando comparada à forma individual|(17).

Os mesmos autores reforçam em outro estudo que a IB é uma estratégia efetiva, pautada nos estágios de motivação do cliente, para que assim se obtenha uma mudança comportamental do modo de beber, sugerindo a aplicação da técnica em diferentes locais, no cotidiano do trabalho dos enfermeiros dos serviços de atenção primária, integrando a IB às consultas de enfermagem ou grupos de psicoeducação existentes nos serviços de saúde ${ }^{(18)}$.

Contextualizando dados epidemiológicos, uma pesquisa que objetivou avaliar a Intervenção Breve em utentes de unidade de saúde familiar mostrou que $88,6 \%$ dos utentes encontravam-se em nível de risco I e 11,4\% no nível de risco II. E no seguimento de cinco meses após a IB, 97,7\% encontravam-se em nível de risco I, e 3\% no nível de risco II, concluindo que as IBs tiveram efeito na diminuição e estabilização nos níveis de risco de consumo de álcool, reforçando a importância da aplicação de IBss nos cuidados de saúde primários $^{(8)}$.

Outro achado investigativo que também utilizou a eficácia da IB em serviço de urgência apontou que três utentes referenciados para consulta de provável perturbação por uso de álcool, três meses após as IBs, reduziram os níveis de risco(19). Pesquisa que identificou o impacto de uma IB emparelhada com arteterapia em pacientes que usam álcool apresentou uma elevada eficácia na diminuição/cessação do uso abusivo de álcool. A implementação de diversas frentes de atendimento a pessoas com uso abusivo de álcool na rede de atenção primária subsidiam um atendimento adequado e efetivo ao paciente, confirmado pelo número expressivo de indivíduos que cessaram o uso do álcool(20).

Em inquérito que analisou o caso clínico de uma paciente feminina que reporta o consumo de álcool de risco e um alto nível de estresse, realizado no México, os resultados obtidos no final e no seguimento da intervenção mostram que a paciente deixou de consumir álcool e seus níveis de estresse diminuíram ${ }^{(21)}$.

Vale destacar que as IBs também podem ser aplicadas em outros contextos, tal como estudo(12) que realçou o efeito positivo da IB na redução do consumo problemático de álcool em portadores de HIV, mostrando evolução positiva quando comparada ao grupo controle, que recebeu ensinos habituais.

Portanto, diante dos resultados encontrados e da literatura comparada, pode-se evidenciar o efeito positivo da IBs na redução do consumo de risco de álcool, sendo assim confirmada a hipótese incialmente levantada. Por fim, considera-se importante maiores investigações em diversos contextos com pacientes submetidos a esta intervenção, para que assim se reforce a efetividade da IB na redução do consumo de risco/nocivo de álcool.

A amostragem foi não probabilística, com utentes que estavam em atendimentos nos serviços de saúde, podendo assim ter provocado vieses e dificultado a representatividade da população alvo. Além do grupo único, ou seja, sem grupo controle, permitindo assim comprometimento da validade interna dos dados, pois não foram controladas outras variáveis que pudessem garantir a evolução positiva única a partir da IBs, sendo estas as limitações deste estudo.

Outrora, mesmo a avaliação após intervenção ter seguido os protocolos necessários, com um seguimento de quatro meses, seriam válidas avalições posteriores para se verificar a continuidade e manutenção da eficácia das IBs. O segmento realizado serviu para reforçar a importância do rastreio precoce e a instrumentalização das IBs na redução do consumo de risco de álcool, sendo esta a maior potencialidade deste estudo.

Novas pesquisas devem ser realizadas, entretanto devem considerar as limitações existentes, pois, embora as pesquisas mostrem resultados positivos quando submetidos às IBs, sugere-se que em pesquisas futuras sejam integrados grupos controle e aumento do tempo de seguimento, sendo fundamental investir na educação continuada dos 
profissionais da atenção primária para consolidação das melhorias obtidas através das IBs e fortalecimento das evidências.

\section{CONCLUSÃO}

Evidenciou-se efeito positivo das Intervenções Breves na redução de risco de consumo de álcool, sendo estas artifícios essenciais na detecção precoce do consumo de álcool, e os enfermeiros imprescindíveis na identificação e intervenção dos indivíduos que apresentam consumo de risco nocivo de álcool.

É válido salientar a importância da disseminação de treinamentos, capacitação e formação de profissionais, com destaque para os enfermeiros, a fim de implementar as IBs, para detecção, monitorização e encaminhamentos necessários diante de pessoas com uso problemático de álcool. Esta temática é importante no contexto da enfermagem, saúde mental e saúde pública, a fim de fortalecer os cuidados e assistência à saúde de qualidade, pois as IBs são recursos efetivos e simples, que podem ser utilizados principalmente nos cuidados primários de saúde.

Por fim, as implicações para a área da enfermagem são de que é uma prática empreendedora, fomentada em bases científicas, que sobreleva o cuidado de saúde mental e fortalece a prática do enfermeiro na atuação profissional e difunde uma tecnologia de cuidado de baixo custo, capaz de reduzir o consumo de risco/nocivo de álcool e prevenir o uso, evitando desta maneira morbidades e problemas relacionados ao longo do tempo.

\section{REFERÊNCIAS}

1. World Health Organization (WHO). Global Report on Alcohol. [Internt]. Geneva: WHO; 2018. [acesso em 17 set 2019]. Disponível em: https://apps.who.int/iris/rest/bitstreams/1151838/retrieve.

2. Enrique HRV. La promoción de la salud y el papel del maestro em la prevención de adicciones. Atenas, Revista Científico Pedagógica. [Internet]. 2016 [acesso em 04 jan 2020]; 2(34). Disponível em: http:// education.esp.macam.ac.il/article/1418.

3. Relatório Anual: a situação do país em matéria de álcool autor: serviço de intervenção nos comportamentos aditivos e nas dependências: Direção de Serviços de Monitorização e Informação Divisão de Estatística e Investigação. [Internet]. 2018 [acesso em 06 jan 2020]. Disponível em: http://www. sicad.pt.

4. Babor TH, Higgins-Biddle JC, Saunders JB, Monteiro MG. AUDIT, the alcohol use disorders identification test: guidelines for use in primary health care. 12. ed. Genebra: OMS; 2001.

5. Pereira MO, Anginoni BM, Ferreira N da C, Oliveira MAF de, Vargas D de, Colvero L de A. Efetividade da intervenção breve para o uso abusivo de álcool na atenção primária: revisão sistemática. Rev. bras. enferm. [Internet]. 2013 [acesso em 11 nov 2019]; 66(3). Disponível em: https://doi.org/10.1590/S003471672013000300018.

6. Rosa NRP, Abreu AMM, Barroso TMMD de A. Efeito das intervenções breves na redução do consumo de risco nos utentes em tratamento com metadona. Rev. Enf. Ref. [Internet]. 2015 [acesso em $20 \mathrm{abr}$ 2020]; 4(6). Disponível em: http://dx.doi.org/10.12707/RIV14082.

7. Gonçalves AM de S, Santos MA dos, Volpato RMJ, Furtado EF, Barroso TMMD de A, Pillon SC. Atitudes de estudantes de enfermagem frente ao usuário de substâncias e percepções sobre o cuidado religioso/espiritual. Rev Esc Enferm USP. [Internet]. 2018 [acesso em 10 fev 2020]; 52(e03425). Disponível 
em: http://dx.doi.org/10.1590/S1980-220X2017027903425.

8. Jorge FM. Moreira MT, Pereira MO, Barroso TMMD de A. Intervenções breves na redução do consumo de álcool em utentes de uma unidade de saúde familiar. Rev. Enf. Ref. [Internet]. 2017 [acesso em 20 abr 2020]; 4 (14). Disponível em: http://dx.doi.org/10.12707/RIV17037.

9. Barbiani R, Dalla Nora CR, Schaefer R. Nursing pratices in the primary health care context: a scoping review. Rev. Latino-Am. Enfermagem. [Internet]. 2016 [acesso em 20 de dez 2019]; 24(e2721). Disponível em: http://dx.doi.org/10.1590/1518-8345.0880.2721.

10. Abreu AMM, Pereira PMS, Souza MH do N, Barroso TMMD de A. Perfil do consumo de substâncias psicoativas e sua relação com as características sociodemográficas: uma contribuição para intervenção breve na atenção primária à saúde, Rio de Janeiro, Brasil. Texto contexto-enferm. [Internet]. 2016 [acesso em 10 nov 2019]; 25(4). Disponível em: http://dx.doi.org/10.1590/0104-07072016001450015.

11. Direção-Geral da Saúde. Circular Normativa n³0/2012 atualizada a 18 de dezembro de 2014. [Internet]. 2014 [acesso em 17 set 2019]. Disponível em: https://www.dgs.pt/directrizes-da-dgs/normas-ecirculares-normativas/norma-n-0302012-de-28122012-png.aspx.

12. Patrício SM, Finnell DS, Barroso T. Efeito das intervenções breves na redução do consumo de álcool em indivíduos portadores do vírus da imunodeficiência humana. Rev. Enf. Ref. [Internet]. 2016 [acesso 20 dez 2018]; 4 (11). Disponível em: http://dx.doi.org/10.12707/RIV16052.

13. Zerbetto $S R$, Furino $V$ de $O$, Furino $F$ de $O$. A implementação da intervenção breve na atenção primária à saúde: revisão integrativa. Rev. APS. [Internet]. 2017 [acesso em 4 jan 2020]; 20(1). Disponível em: https://doi.org/10.34019/1809-8363.2017.v20.15782.

14. Abreu AMM, Marinho GL, Jomar RT. Necessidade de intervenção breve por uso de álcool, tabaco e outras drogas entre usuários da atenção primária à saúde. J. bras. psiquiatr [Internet]. 2017 [acesso em 29 mar 2020]; 66(2). Disponível em: https://doi.org/10.1590/0047-2085000000157.

15. Silva EC, Tucci AM. Intervenção breve para redução do consumo de álcool e suas consequências em estudantes universitários brasileiros. Psicol. Reflex. Crit [Internet]. 2015 [acesso em 4 jan 2020]; 28(4). Disponível em: https://doi.org/10.1590/1678-7153.201528410.

16. Sawicki WC, Fram DS, Belasco AGS. Intervenção breve aplicada a universitários consumidores de risco de bebidas alcoólicas. SMAD, Rev. Eletrônica Saúde Mental Álcool Drog. [Internet]. 2018 [acesso em 5 fev 2020]; 14(4). Disponível em: http://dx.doi.org/10.11606/issn.1806-6976.smad.2018.000414.

17. Soares J, Vargas D de. Efetividade da intervenção breve grupal no uso nocivo de álcool na atenção primária à saúde. Rev Saude Publica. [Internet] 2019 [acesso em 29 mar 2020]; 53(2). Disponível em: https://doi.org/10.11606/S1518-8787.2019053000498.

18. Soares J, Vargas D de. Intervenção breve grupal: efetividade na motivação para a mudança do uso de álcool. Rev bras enferm. [Internet]. 2020 [acesso em 30 mar 2020]; 73(1). Disponível em: https://doi. org/10.1590/0034-7167-2018-0138.

19. Esgaio TMG, Barroso TMMD de A. Screening e intervenções breves na redução do consumo de álcool em utentes num serviço de urgência. Rev. Enf. Ref. [Internet]. 2018 [acesso em 19 dez 2019]; IV(17). Disponível em: http://dx.doi.org/10.12707/RIV17110.

20. Soares MH, Rolin TF de C, Machado FP, Ramos LKF, Rampazzo ARP. Impacto da intervenção breve e arteterapia para usuários de álcool. Rev bras enferm. [Internet]. 2019 [acesso em 20 mar 2020]; 72(6). Disponível em: http://dx.doi.org/10.1590/0034-7167-2018-0317.

21. Ocampo LF, Castillo MMA, Lázaro AM. Reducción del consumo de alcohol mediante mindfulness y consejo breve. J. Health NPEPS [Internet]. 2018 [acesso em 2 mar 2020]; 3(1). Disponível em: https:// periodicos.unemat.br/index.php/jhnpeps/article/view/2800/2365. 
Recebido: 07/05/2020

Finalizado: 22/09/2020

Editora associada: Luciana Puchalski Kalinke

Autor Correspondente:

Fernanda Matos Fernandes Castelo Branco

Universidade Federal do Amapá

Rd. BR 156, 3051 - 68980-000 - Oiapoque, Amapá, Brasil

E-mail: fmfernandescb@gmail.com

Contribuição dos autores:

Contribuições substanciais para a concepção ou desenho do estudo; ou a aquisição, análise ou interpretação de dados do estudo - FMFCB, ACPAF, TMMDAB

Elaboração e revisão crítica do conteúdo intelectual do estudo - FMFCB, ACPAF, TMMDAB

Aprovação da versão final do estudo a ser publicado - FMFCB, ACPAF, TMMDAB

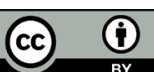

Este obra está licenciado com uma Licença Creative Commons Atribuição 4.0 Internacional. 